\title{
Longitudinal Tractography with Application to Neuronal Fiber Trajectory Reconstruction in Neonates
}

\author{
Pew-Thian Yap ${ }^{1}$, John H. Gilmore ${ }^{2}$, Weili Lin ${ }^{1}$, and Dinggang Shen ${ }^{1}$ \\ 1 BRIC, Department of Radiology and \\ 2 Department of Pyschiatry \\ University of North Carolina at Chapel Hill, NC \\ \{ptyap,jgilmore, weili_lin, dgshen\}@med.unc.edu
}

\begin{abstract}
This paper presents a novel tractography algorithm for more accurate reconstruction of fiber trajectories in low SNR diffusion-weighted images, such as neonatal scans. We leverage information from a latertime-point longitudinal scan to obtain more reliable estimates of local fiber orientations. Specifically, we determine the orientation posterior probability at each voxel location by utilizing prior information given by the longitudinal scan, and with the likelihood function formulated based on the Watson distribution. We incorporate this Bayesian model of local orientations into a state-space model for particle-filtering-based probabilistic tracking, catering for the possibility of crossing fibers by modeling multiple orientations per voxel. Regularity of fibers is enforced by encouraging smooth transitions of orientations in subsequent locations traversed by the fiber. Experimental results performed on neonatal scans indicate that fiber reconstruction is significantly improved with less stray fibers and is closer to what one would expect anatomically.
\end{abstract}

\section{Introduction}

The human brain is a complex system that is capable of integrating massive amount of information with startling efficiency. A comprehensive description of the architecture of the anatomical connectivity patterns is therefore fundamentally important in cognitive neuroscience and neuropsychology, as it reveals how functional brain states emerge from their underlying structural substrates and provides new mechanistic insights into the association of brain functional deficits with the underlying structural disruption [1.

The neonatal brain provides a window for insights into perhaps the most important phase of human brain development. To this end, diffusion-weighted imaging (DWI) plays an indispensable role in the in vivo characterization of brain structural circuity, which relates different functional regions. DWI-based connectivity analysis of neonates, however, is often impeded by the unreliability of neuronal fiber trajectory reconstruction due to the often lower quality of the diffusion-weighted scans. Noisy estimates of local fiber orientations can propagate and accummultate in the course of trajectory reconstruction, especially if 
the tractography algorithm is greedy in nature, rendering the validity of subsequent tract-based analysis questionable. We present in this paper a remedy to this problem by leveraging prior information from longitudinal scans to improve the accuracy of local fiber orientation estimates for more accurate trajectory reconstruction of the neuronal fibers.

Cook et al. 22 proposed an atlas based approach for better tractography outcome by modifying the stochastic white matter tractography algorithm developed by Friman et al. [3. An atlas was first generated by computing for each voxel the dyadic tensor of the principal directions from a set of diffusion tensor images. The atlas encapsulates the mean local fiber orientations as well as their degrees of orientation dispersion, serving as prior information to the Bayesian stochastic tractography framework in [3]. This approach, while effective, has two shortcomings: 1) Its formulation is limited to one orientation per voxel, seriously limiting the ability of the algorithm in accounting for fiber crossing, branching or kissing, and 2) The atlas is not subject-specific, causing loss of fiber tracts in regions where the subject and the atlas disagree.

The contributions of this paper are: 1) The formulation and evaluation of a fiber tractography algorithm that is guided by longitudinal prior information for more robust fiber trajectory reconstruction, especially in images with lower SNR, and 2) The evaluation of how modeling multiple local fiber orientations improves tractography outcome in regions with complex diffusion architecture.

\section{Approach}

\subsection{Modeling Local Fiber Orientations}

A white matter fiber can be modeled as a finite-length path parameterized by a train of unit length vectors. We use the following notation for such a path: $\mathbf{v}_{(1: T)}=\left\{\mathbf{v}_{(1)}, \ldots, \mathbf{v}_{(T)}\right\}$. We further assume that a fiber path can be traced by tracking the trajectory of a particle traveling in an orientation field. Each particle is endowed with an initial speed in an appropriate direction. It then moves with constant speed to position $\mathbf{x}_{(t)}$ according to

$$
\mathbf{x}_{(t+1)}=\mathbf{x}_{(t)}+s \mathbf{v}_{(t)}
$$

where $t$ is the time index and $s$ is the step length. To reconstruct the fiber trajectories, we need to determine the probability density function (PDF) of the local fiber orientation $f\left(\mathbf{v}_{(t)} \mid \mathbf{v}_{(t-1)}, \boldsymbol{\theta}_{(t)}, \boldsymbol{\theta}_{(t)}^{\prime}, D_{(t)}\right)$. Variable $D_{(t)}=1, \ldots, \Omega$ is the orientation index; each voxel may contain up to $\Omega$ orientations, as in the case of high angular resolution diffusion imaging (HARDI). Sets $\boldsymbol{\theta}_{(t)}$ and $\boldsymbol{\theta}_{(t)}^{\prime}$ are collections of orientations and their strengths in the neighborhood of $\mathbf{x}_{(t)}$ of the neonatal and longitudinal scans, respectively. Specifically, we define a neighborhood $\mathcal{N}\left(\mathbf{x}_{(t)}\right)$ (e.g., a $3 \times 3 \times 3$ neighborhood) in the vicinity of $\mathbf{x}_{(t)}$ and, for each voxel $i$ in the neighborhood, collect all corresponding orientations $\mathbf{v}_{(t), i}^{\left[D_{(t)}\right]}$ and their strengths $\rho_{(t), i}^{\left[D_{(t)}\right]}$ (e.g., magnitudes of the orientation distribution functions at corresponding orientations), spatially weighted by a Gaussian kernel 
so that voxels further away from the neighborhood center are deemphasized. The orientations are first sorted to avoid the bias discussed in 4. Applying Bayes' theorem, we have

$$
f\left(\mathbf{v}_{(t)} \mid \mathbf{v}_{(t-1)}, \boldsymbol{\theta}_{(t)}, \boldsymbol{\theta}_{(t)}^{\prime}, D_{(t)}\right)=\frac{f\left(\boldsymbol{\theta}_{(t)} \mid \mathbf{v}_{(t)}, \mathbf{v}_{(t-1)}, \boldsymbol{\theta}_{(t)}^{\prime}, D_{(t)}\right) f\left(\mathbf{v}_{(t)} \mid \mathbf{v}_{(t-1)}, \boldsymbol{\theta}_{(t)}^{\prime}, D_{(t)}\right)}{f\left(\boldsymbol{\theta}_{(t)} \mid \mathbf{v}_{(t-1)}, \boldsymbol{\theta}_{(t)}^{\prime}, D_{(t)}\right)} .
$$

Since the orientations in both images are estimated independently in a voxel-wise fashion, $f\left(\boldsymbol{\theta}_{(t)} \mid \mathbf{v}_{(t)}, \mathbf{v}_{(t-1)}, \boldsymbol{\theta}_{(t)}^{\prime}, D_{(t)}\right) \quad=\quad f\left(\boldsymbol{\theta}_{(t)} \mid \mathbf{v}_{(t)}, D_{(t)}\right) \quad$ and $f\left(\boldsymbol{\theta}_{(t)} \mid \mathbf{v}_{(t-1)}, \boldsymbol{\theta}_{(t)}^{\prime}, D_{(t)}\right)$

$=f\left(\boldsymbol{\theta}_{(t)} \mid D_{(t)}\right)$. The equation can then be written as

$$
f\left(\mathbf{v}_{(t)} \mid \mathbf{v}_{(t-1)}, \boldsymbol{\theta}_{(t)}, \boldsymbol{\theta}_{(t)}^{\prime}, D_{(t)}\right)=\frac{f\left(\boldsymbol{\theta}_{(t)} \mid \mathbf{v}_{(t)}, D_{(t)}\right) f\left(\mathbf{v}_{(t)} \mid \mathbf{v}_{(t-1)}, \boldsymbol{\theta}_{(t)}^{\prime}, D_{(t)}\right)}{f\left(\boldsymbol{\theta}_{(t)} \mid D_{(t)}\right)} .
$$

The factor $f\left(\boldsymbol{\theta}_{(t)} \mid D_{(t)}\right)$ normalizes the posterior probability function to have a unit volume and can thus be written as the integral of the numerator

$$
f\left(\boldsymbol{\theta}_{(t)} \mid D_{(t)}\right)=\int_{\mathbf{v}_{(t)}, \mathbf{v}_{(t-1)}, \boldsymbol{\theta}_{(t)}^{\prime}} f\left(\boldsymbol{\theta}_{(t)} \mid \mathbf{v}_{(t)}, D_{(t)}\right) f\left(\mathbf{v}_{(t)} \mid \mathbf{v}_{(t-1)}, \boldsymbol{\theta}_{(t)}^{\prime}, D_{(t)}\right) d \mathbf{v}_{(t)} d \mathbf{v}_{(t-1)} d \boldsymbol{\theta}_{(t)}^{\prime} .
$$

In what follows, we will discuss how the likelihood $f\left(\boldsymbol{\theta}_{(t)} \mid \mathbf{v}_{(t)}, D_{(t)}\right)$ and prior $f\left(\mathbf{v}_{(t)} \mid \mathbf{v}_{(t-1)}, \boldsymbol{\theta}_{(t)}^{\prime}, D_{(t)}\right)$ can be computed.

Likelihood: We assume that the orientations observed in the neighborhood of $\mathbf{x}_{(t)}$ can be regarded as noisy observations of $\mathbf{v}_{(t)}$, characterized by the Watson distribution with probability density function (PDF) [5]

$$
f(\mathbf{w} \mid \boldsymbol{\mu}, \kappa)=C(\kappa) \mathrm{e}^{\kappa\left(\mathbf{w}^{\mathrm{T}} \boldsymbol{\mu}\right)^{2}} .
$$

The parameter $\boldsymbol{\mu}$ is a unit vector called the mean orientation and $\kappa$ is a positive constant called the concentration parameter. The squared exponential in (5) ensures that the distribution is antipodal symmetric. The density has maxima at $\pm \boldsymbol{\mu}$ and becomes more concentrated around $\pm \boldsymbol{\mu}$ as $\kappa$ increases. The density is also rotationally invariant around $\pm \boldsymbol{\mu} . C(\kappa)$ is a normalizing constant to ensure that the density function integrates to unity over the unit sphere. By letting $\boldsymbol{\mu}=\mathbf{v}_{(t)}$, the joint distribution, or the likelihood, of the observed orientations $\boldsymbol{\theta}_{(t)}$ can be written as

$$
f\left(\boldsymbol{\theta}_{(t)} \mid \mathbf{v}_{(t)}, D_{(t)}\right)=\prod_{(\mathbf{w}, \rho) \in \boldsymbol{\theta}_{(t)}^{[D(t)}} C(\kappa) e^{\rho \kappa\left(\mathbf{w}^{\mathrm{T}} \mathbf{v}_{(t)}\right)^{2}}
$$

where $\boldsymbol{\theta}_{(t)}^{\left[D_{(t)}\right]}$ is a subset of $\boldsymbol{\theta}_{(t)}$ consisting only of the group of orientations specified by $D_{(t)}$. This equation is substituted into (3) to obtain the posterior distribution. 
Priors: Via the probability function $f\left(\mathbf{v}_{(t)} \mid \mathbf{v}_{(t-1)}, \boldsymbol{\theta}_{(t)}^{\prime}, D_{(t)}\right)$, we encode our prior knowledge about fiber regularity and about orientation information from the longitudinal scan. We define the prior probability function as

$$
\begin{aligned}
& f\left(\mathbf{v}_{(t)} \mid \mathbf{v}_{(t-1)}, \boldsymbol{\theta}_{(t)}^{\prime}, D_{(t)}\right) \propto
\end{aligned}
$$

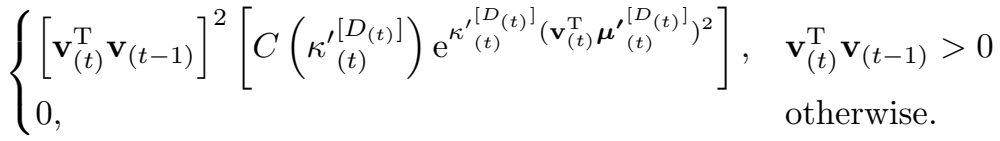

The first term on the right enforces the regularity constraint during fiber reconstruction. The second term transfuses orientation information from the longitudinal scan. The Watson distribution is used to represent the distribution of the orientations in $\boldsymbol{\theta}_{(t)}\left[D_{(t)}\right]$, with mean orientation $\boldsymbol{\mu}_{(t)}{ }_{\left(D_{(t)}\right]}^{\left[D^{2}\right.}$ and concentration parameter $\kappa^{\prime\left[D_{(t)}\right]}$. Maximum likelihood estimates of the these parameters can be obtained using the method described in [5].

\subsection{State-Space Model}

Tractography is assumed to be a stochastic process that can be represented using a state-space model with the local fiber orientation $\mathbf{v}_{(t)}$ as the observation, and the orientation index $D_{(t)}$ as the state. Given the previous oriention $\mathbf{v}_{(t-1)}$ and the current state $D_{(t)}$, the observation probability is defined according to (3). Noting that the posterior probability for selection of a particular value of the orientation index $D_{(t)}$ is

$$
f\left(D_{(t)} \mid \mathbf{v}_{(t-1)}, \boldsymbol{\theta}_{(t)}, \boldsymbol{\theta}_{(t)}^{\prime}\right)=\frac{f\left(\boldsymbol{\theta}_{(t)} \mid \mathbf{v}_{(t-1)}, \boldsymbol{\theta}_{(t)}^{\prime}, D_{(t)}\right) f\left(D_{(t)} \mid \mathbf{v}_{(t-1)}, \boldsymbol{\theta}_{(t)}^{\prime}\right)}{f\left(\boldsymbol{\theta}_{(t)} \mid \mathbf{v}_{(t-1)}, \boldsymbol{\theta}_{(t)}^{\prime}\right)}
$$

and by letting

$$
f\left(\boldsymbol{\theta}_{(t)} \mid \mathbf{v}_{(t-1)}, \boldsymbol{\theta}_{(t)}^{\prime}, D_{(t)}\right) \propto \bar{\rho}_{(t)}^{\left[D_{(t)}\right]}, \quad f\left(D_{(t)} \mid \mathbf{v}_{(t-1)}, \boldsymbol{\theta}_{(t)}^{\prime}\right) \propto\left(\mathbf{v}_{(t-1)}^{\mathrm{T}} \boldsymbol{\mu}_{(t)}^{\left.\prime D_{(t)}\right]}\right)^{2},
$$

the transition probability can be defined as

$$
f\left(D_{(t)} \mid \mathbf{v}_{(t-1)}, \boldsymbol{\theta}_{(t)}, \boldsymbol{\theta}_{(t)}^{\prime}\right) \propto \bar{\rho}_{(t)}^{\left[D_{(t)}\right]}\left(\mathbf{v}_{(t-1)}^{\mathrm{T}} \boldsymbol{\mu}_{(t)}^{\prime\left[D_{(t)}\right]}\right)^{2} .
$$

where $\bar{\rho}_{(t)}^{\left[D_{(t)}\right]}$ denotes the average strength of orientations in $\boldsymbol{\theta}_{(t)}^{\left[D_{(t)}\right]}$. For sequential sampling of fiber paths, we need to draw random samples of local fiber orientations from the observation probability (3) and transition probability (10). For drawing samples from complicated and high dimensional PDFs, one can always resort to Markov Chain Monte Carlo (MCMC) techniques. The probability of a path of a given length $T$ is

$$
\begin{aligned}
f\left(\mathbf{v}_{(1: T)} \mid \boldsymbol{\theta}_{(1: T)}, \boldsymbol{\theta}_{(1: T)}^{\prime}\right)=f\left(D_{(1)}\right) f\left(\mathbf{v}_{(1)} \mid \mathbf{v}_{(1)}, \boldsymbol{\theta}_{(1)}, \boldsymbol{\theta}_{(1)}^{\prime}, D_{(1)}\right) \times \\
\Pi_{t=2}^{T} f\left(D_{(t)} \mid \mathbf{v}_{(t-1)}, \boldsymbol{\theta}_{(t)}, \boldsymbol{\theta}_{(t)}^{\prime}\right) f\left(\mathbf{v}_{(t)} \mid \mathbf{v}_{(t-1)}, \boldsymbol{\theta}_{(t)}, \boldsymbol{\theta}_{(t)}^{\prime}, D_{(t)}\right) .
\end{aligned}
$$


Tracking is stopped if the trajectory reaches a voxel with orientation coherence

$$
\beta^{\left[D_{(t)}\right]}=1-\frac{1}{2}\left\{\sqrt{\frac{\lambda_{2}^{\left[D_{(t)}\right]}+\lambda_{3}^{\left[D_{(t)}\right]}}{2 \lambda_{1}^{\left[D_{(t)}\right]}}}+\sqrt{\frac{{\lambda^{\prime}}_{2}^{\left[D_{(t)}\right]}+{\lambda^{\prime}}_{3}^{\left[D_{(t)}\right]}}{2{\lambda^{\prime}}_{1}^{\left[D_{(t)}\right]}}}\right\}, \quad \beta^{\left[D_{(t)}\right]} \in[0,1],
$$

falling below a predefined threshold $\beta_{0}$, or simply when the brain boundary is encountered. The $\lambda$ 's are the eigenvalues of the dyadic tensors computed from $\boldsymbol{\theta}_{(t)}^{\left[D_{(t)}\right]}$ and $\boldsymbol{\theta}_{(t)}^{{ }^{\left[D_{(t)}\right]}}$ [2, 4]. Perfect alignment of the orientations indicated by $D_{(t)}$ results in $\beta^{\left[D_{(t)}\right]}=1$ and an uniform distribution of orientations results in $\beta^{\left[D_{(t)}\right]}=0$.

\section{Results}

\subsection{Materials}

Diffusion-weighted images of 10 infants were acquired at two time points: one month and one year after birth. Diffusion gradients were applied in 42 noncollinear directions with diffusion weighting $b=1000 \mathrm{~s} / \mathrm{mm}^{2}$, repetition time $(\mathrm{TR})=7,680 \mathrm{~ms}$ and echo time $(\mathrm{TE})=82 \mathrm{~ms}$. The scans covered the whole brain with a resolution of $2 \times 2 \times 2 \mathrm{~mm}^{3}$. Data post-processing includes brain skull removal, motion correction and eddy current correction using algorithms developed and distributed as part of the FMRIB Software Library (FSL) package. Each neonatal scan was co-registered with their respective longitudinal scan so that they reside in a common space.

\subsection{Tractography}

To evaluate the effectiveness of the proposed method, we performed tractography based on seeds placed at the points where the midline crosses the splenium of the corpus callosum. 3000 trajectories were initiated from each seed point. The step size was fixed at $1 \mathrm{~mm}$. The maximum allowable orientation coherence was set to 0.1 . Various configurations were used:

(a) Tractography on the neonatal scan alone with no prior information from the longitudinal scan.

(b) Tractography on the neonatal scan guided by prior information from the longitudinal scan.

(c) Tractograph on the longitudinal scan.

(d) Tractography on the neonatal scan, using only the first principal orientation with no prior information.

(e) Tractography on the neonatal scan guided by longitudinal prior information from the longitudinal scan, using only the first principal orientations.

(f) Tractography on the longitudinal scan using only the first principal orientations. 


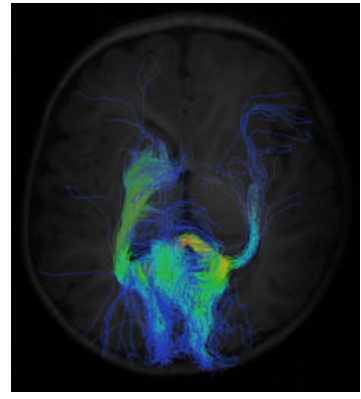

(a) Neonatal Scan

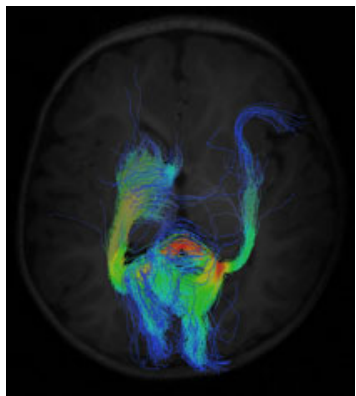

(d) Neonatal Scan - Single Orientation

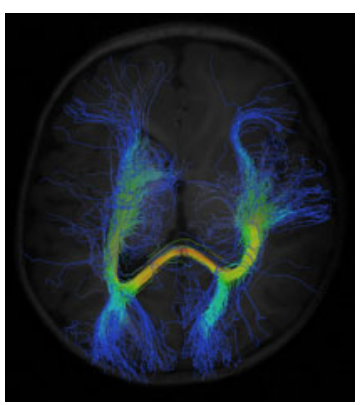

(b) Guided

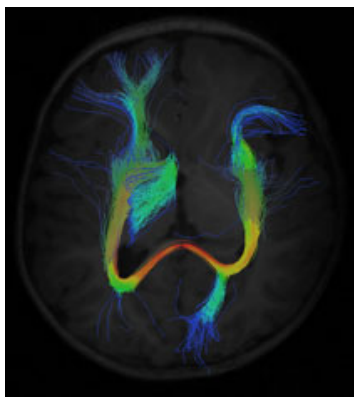

(e) Guided - Single Orientation

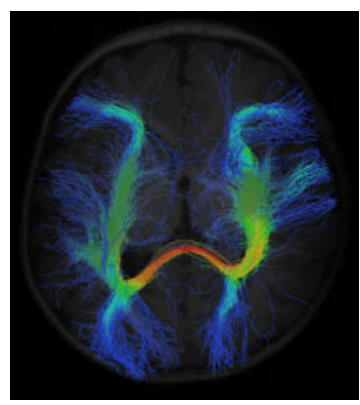

(c) Longitudinal Scan

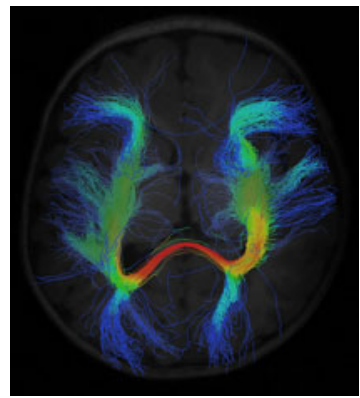

(f) Longitudinal Scan Single Orientation

Fig. 1. Comparison of different tractography schemes. (a) Neonatal scan alone, (b) Neonatal scan + longitudinal scan, (c) Longitudinal scan alone, (d) Neonatal scan alone (single orientation), (e) Neonatal scan + longitudinal scan (single orientation), and (f) Longitudinal scan alone (single orientation).

The respective results, shown in Fig. 1, indicate that the proposed method (Fig. 1(b)) gives reasonable results. Tractography, when performed based on orientation information given by the neonatal scan alone, results in noisy fiber tracts (Fig. 1(a) and Fig. 1(d)). This is not surprising since neonatal scans typically suffer from lower SNR. The results were improved remarkably by employing prior information given by the longitudinal scan (Fig. 1(c) and Fig. 1(f)). The reconstructed trajectories are generally cleaner with less stray fibers and are in higher agreement with our anatomical understanding of the fibers. Note that by allowing only one orientation per voxel, major fibers are lost. Fig. 11(e) shows that, despite with longitudinal guidance, fibers connecting one of the occipital lobes cannot be correctly preserved and reconstructed.

\subsection{Single- and Multi-orientation Schemes}

For quantification of the effect of single-orientation and multi-orientation schemes on the reconstructed trajectories, various measures were employed. First, we 


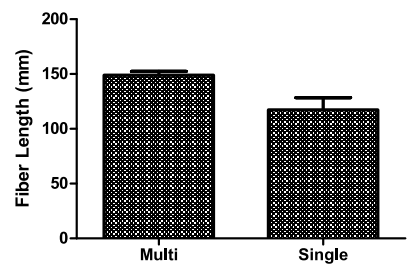

(a) Fiber Length

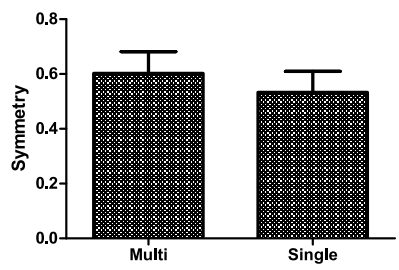

(b) Symmetry

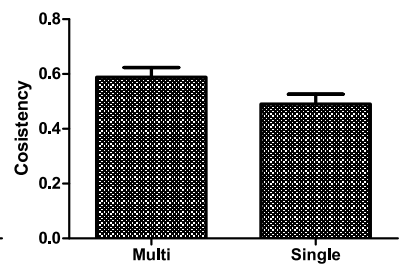

(c) Consistency

Fig. 2. Comparison between the multi- and single-orientation schemes using different statistics. Each bar indicates the mean value, and the error bar indicates the corresponding standard error.

compared the average length of the reconstructed trajectories. A longer length average generally indicates that fiber reconstruction is less likely to be terminated prematurely, possibly due to noisy local fiber orientations. Fig. 2(a) shows that the proposed method results in on average longer trajectories, hinting that by allowing more than one orientation per voxel results in less chances of premature termination at fiber crossings.

Anatomical scans (e.g., T1-weighted and T2-weighted images) of the brain indicate that the brain is mostly symmetrical between the hemispheres. Therefore, one would expect that, to be anatomically sound, the reconstructed trajectories should exhibit some degree of symmetry. For our purpose, we evaluated the degree of symmetry of the reconstructed trajectories by computing the degree of correlation of the connectivity matrices derived from the fibers between the left and right hemispheres. Specifically, this was done by parcellating the brain into 116 regions according to the Automated Anatomical Labelling (AAL) atlas [6], computing the connectivity matrix for each hemisphere based only on the $(116 / 2$ $=58$ ) ROIs in that particular hemisphere and then computing the normalized scalar product of the connectivity matrices of both hemispheres. Each element of the $(58 \times 58)$ connectivity matrix records the number of fibers connecting a pair of ROIs. The results, shown in Fig. 2(b), indicates the multi-orientation scheme yields higher inter-hemispheric consistency compared with the single-orientation scheme, again validating that modeling complex orientation information can be conducive to more accurate reconstruction of fiber trajectories.

Major fiber bundles known to exist in all subjects can be used to test fiber tracking consistency across subjects. Since the seeds used in our evaluation were placed on a major white major structure, we expect the reconstructed trajectories to be relatively consistent across subjects. We tested this by using a similar methodology described in the previous paragraph. But instead of generating the connectivity matrix for each hemisphere separately, we generated a whole brain connectivitiy matrix by considering both hemispheres at the same time. We then computed the mean connectivity matrix over all the 10 subjects. The connectivity matrix of each subject was then compared with this mean matrix (via normalized scalar product) as an indicator of consistency. The higher the 
similarity of all individual connectivity matrices with the mean matrix, the greater is the consistency. Fig. 2(c) indicates that greater consistency can be achieved using the multi-orientation scheme, again validating the effectivenes of the proposed method.

\section{Conclusion}

We have presented a tractography algorihtm that caters especially for low SNR diffusion-weighted images by leveraging prior information from the respective longitudinal scans. Experimental results performed using neonatal scans indicate that the proposed method yields fiber trajectories that are more consistent with our anatomical knowledge. The ability of taking into account multi-orientation information gives further improvement over previous methods by allowing more accurate modeling of complex white matter architecture involving crossing fibers.

Acknowledgment. This work was supported in part by NIH grants: EB006733, EB008374, EB009634, MH088520, HD05300, MH064065, and NS055754.

\section{References}

1. Sporns, O., Tononi, G., Kötter, R.: The human connectome: A structural description of the human brains. PLoS Computational Biology 1(4), e42 (2005)

2. Cook, P.A., Zhang, H., Awate, S.P., Gee, J.C.: Atlas-guided probabilistic diffusiontensor fiber tractography. In: IEEE International Symposium on Biomedical Imaging (ISBI 2008), pp. 951-954 (2008)

3. Friman, O., Farnebäck, G., Westin, C.F.: A bayesian approach for stochastic white matter tractography. IEEE Transactions on Medical Imaging 25, 965-977 (2006)

4. Basser, P.J., Pajevic, S.: Statistical artifacts in diffusion tensor MRI (DT-MRI) caused by background noise. Magnetic Resonance in Medicine 44, 41-50 (2000)

5. Schwartzman, A., Dougherty, R.F., Taylor, J.E.: False discovery rate analysis of brain diffusion direction maps. Annals of Applied Statistics 2(1), 153-175 (2008)

6. Tzourio-Mazoyer, N., Landeau, B., Papathanassiou, D., Crivello, F., Etard, O., Delcroix, N., Mazoyer, B., Joliot, M.: Automated anatomical labeling of activations in SPM using a macroscopic anatomical parcellation of the MNI MRI single-subject brain. Neuroimage 15, 273-289 (2002) 